\title{
Efecto de diferentes dosis de dos enmiendas orgánicas en los componentes de rendimiento en lechuga (Lactuca sativa) ${ }^{24}$
}

\section{Effect of different doses of two organic amendments in the components of performance, in lettuce (lactuca sativa)}

\author{
Rosanna Britos; Hugo Valiente; Beatriz Gómez; Marcos Vega ${ }^{25}$; Danny \\ Ríos $^{26}$; Arturo Estigarribia ${ }^{27}$
}

\section{DOI: https://doi.org/10.36003/Rev.investig.cient.tecnol.V2N2(2018)6}

\section{RESUMEN}

La lechuga (Lactuca sativa), es la hortaliza de hoja más cultivada y consumida en el Paraguay, se considera exigente de nutrientes porque requiere cantidades relativamente grandes en un período de tiempo relativamente corto. Con el objetivo de evaluar el efecto de diferentes dosis de dos enmiendas orgánicas, estiércol vacuno y humus de lombriz en la producción, fue desarrollado en el Instituto Paraguayo de Tecnología Agraria (IPTA) - Caacupé, un experimento utilizando un diseño en bloques completos al azar, con ocho tratamientos y tres repeticiones. Los tratamientos fueron: a) Humus de lombriz T1 $=0 \mathrm{~kg}$ $\mathrm{m}-2, \mathrm{~T} 2=0,4 \mathrm{~kg} \mathrm{~m}-2, \mathrm{~T} 3=0,6 \mathrm{~kg} \mathrm{~m}-2, \mathrm{~T} 4=0,8 \mathrm{~kg} \mathrm{~m}-2 ; \mathrm{b}$ ) Estiércol vacuno T5 $=0 \mathrm{~kg}$ $\mathrm{m}-2, \mathrm{~T} 6=2 \mathrm{~kg} \mathrm{~m}-2, \mathrm{~T} 7=4 \mathrm{~kg} \mathrm{~m}-2$ y T $8=6 \mathrm{~kg} \mathrm{~m}-2$. El material genético utilizado fue la lechuga de la variedad Graciela (tipo Pirati). La superficie de cada unidad experimental fue de $2 \mathrm{~m} 2$, con 4 hileras de lechuga por cada unidad, con un distanciamiento de 0.25 $\mathrm{m}$ entre hileras y $0.25 \mathrm{~m}$ entre plantas. La distancia entre unidades experimentales fue de $0.50 \mathrm{~m}$. La superficie total del experimento fue de $78 \mathrm{~m} 2$ con 864 plantas. Las variables evaluadas fueron altura de planta, masa fresca comercial, masa fresca de hojas, masa seca, número de hojas, peso fresco radicular y peso seco radicular. Los datos fueron sometidos al análisis de varianza (ANAVA), evaluando las diferencias entre medias por la prueba de Tukey al $5 \%$ de probabilidad de error. Los resultados obtenidos indican que la aplicación de humus de lombriz y estiércol vacuno no ejerció ningún efecto sobre los componentes de rendimiento en un ciclo de cultivo de lechuga.

Palabras clave: Lactuca sativa; Humus de lombriz; Estiércol vacuno; Dosis

\footnotetext{
${ }^{24}$ Fecha de recepción: septiembre 2018; fecha de aceptación: octubre 2018

${ }^{25}$ Técnicos del Instituto Paraguayo de Tecnología Agraria, Caacupé, Paraguay.

${ }^{26}$ Estudiante de la Maestría en Ciencia del Suelo y Ordenamiento Territorial de la Facultad de Ciencias Agrarias de la Universidad Nacional de Asunción, San Lorenzo, Paraguay.

${ }^{27}$ Egresado de la Facultad de Ciencias Agrarias de la Universidad Nacional de Asunción, San Lorenzo , Paraguay
} 


\section{ABSTRACT}

Lettuce (Lactuca sativa), the most cultivated and consumed leaf vegetable in Paraguay, is considered nutrient demanding because it requires relatively large quantities in a relatively short period of time. In order to evaluate the effect of different doses of two organic amendments, cattle manure and earthworm humus in production, it was developed in the Paraguayan Institute of Agricultural Technology (IPTA) - Caacupé, Paraguay, an experiment using a randomized complete block design, with eight treatments and three repetitions. The treatments were: a) Worm humus $\mathrm{T} 1=0 \mathrm{~kg} \mathrm{~m}-2, \mathrm{~T} 2=0.4 \mathrm{~kg} \mathrm{~m}-2, \mathrm{~T} 3=$ $0.6 \mathrm{~kg} \mathrm{~m}-2, \mathrm{~T} 4=0.8 \mathrm{~kg} \mathrm{~m}-2 ; \mathrm{b})$ Cattle manure $\mathrm{T} 5=0 \mathrm{~kg} \mathrm{~m}-2, \mathrm{~T} 6=2 \mathrm{~kg} \mathrm{~m}-2, \mathrm{~T} 7=4 \mathrm{~kg}$ $\mathrm{m}-2$ and $\mathrm{T} 8=6 \mathrm{~kg} \mathrm{~m}-2$. The genetic material used was the lettuce of the Graciela variety (Pirati type). The surface of each experimental unit was $2 \mathrm{~m} 2$, with 4 rows of lettuce per unit, with a distance of $0.25 \mathrm{~m}$ between rows and $0.25 \mathrm{~m}$ between plants. The distance between experimental units was $0.50 \mathrm{~m}$. The total area of the experiment was $78 \mathrm{~m} 2$ with 864 plants. The variables evaluated were plant height, commercial fresh mass, fresh leaf mass, dry mass, number of leaves, fresh root weight and dry root weight. The data were subjected to analysis of variance (ANAVA), evaluating the differences between means by the Tukey test at $5 \%$ error probability. The results obtained indicate that the application of worm humus and cattle manure did not exert any effect on the yield components in a lettuce crop cycle.

Keywords: Lactuca sativa; Worm humus; Cattle manure; Dose 


\section{INTRODUCCIÓN}

Las hortalizas de hoja, así como otras verduras, se consideran exigentes de nutrientes porque requieren cantidades relativamente grandes en un período de tiempo relativamente corto (Grangeiro et al., 2006). Por lo tanto, las aplicaciones de altas tasas de fertilizantes orgánicos y minerales son comunes en el cultivo de verduras de hoja (Pôrto et al., 2008; Santi et al., 2010).

Entre las hortalizas de hoja, la lechuga (Lactuca sativa) es la más cultivada y consumida en Paraguay, y el consumo y la superficie plantada de lechuga en particular ha aumentado considerablemente en los últimos años (Dirección de Censos y Estadísticas Agropecuarias, 2016).

La fertilización orgánica es frecuentemente utilizada por los productores de vegetales, y las tasas aplicadas dependen, entre otros factores, del tipo y la composición química del fertilizante orgánico, la textura del suelo y la fertilidad inicial (Silva, 2008). Entre los fertilizantes orgánicos utilizados para el cultivo de hortalizas, el estiércol vacuno es el más común. Los fertilizantes orgánicos mejoran las propiedades químicas, físicas y biológicas del suelo, lo que contribuye a elevar los rendimientos de los cultivos (Melo et al., 2008).

Desde el punto de vista químico, el uso de fertilizantes orgánicos como el estiércol del ganado, mantiene o aumenta el contenido de materia orgánica del suelo y proporciona a las plantas nutrientes (Batista et al., 2012). Sin embargo, las tasas muy altas pueden alterar el equilibrio entre los nutrientes y aumentar el contenido de sal en el suelo, lo que puede obstaculizar los rendimientos de vegetales. Este problema suele ser mayor en suelos arenosos, donde los fertilizantes orgánicos se descomponen más rápidamente y los contenidos de sal en la solución del suelo son más altos (Fio- reze et al., 2012).

La producción de verduras de hoja, se cultivan durante todo el año en la misma área, generalmente con la aplicación de fertilizantes orgánicos antes de la siembra de cada cultivo.

El humus de lombriz es un material estabilizado, libre de olores y con características deseables como componente de sustratos de cultivo distintos del suelo, que se obtiene por la descomposición de residuos orgánicos a partir de la acción conjunta de las lombrices de tierra (Eisenia foetida) y distintos microorganismos (Paul \& Metzger, 2005; Melgar-Ramírez \& Pascual-Alex, 2010; Basheer \& Agrawal, 2013).

Entre sus características fundamentales se encuentran su buena estructura, drenaje, aireación, capacidad de retención de humedad, abundante cantidad de nutrientes en formas fácilmente asimilables para las plantas; además de la presencia de partículas cuya área superficial favorece la retención de los nutrientes, y provee micrositios aptos para la actividad de especies microbianas con comprobada aptitud para la generación de sustancias con acción promotora del crecimiento, del tipo de las auxinas, giberelinas y ácidos húmicos (Aalok et al., 2008 ;Argüello et al., 2013).

La incorporación de humus de lombriz a las mezclas de siembra favorece el crecimiento y producción en diversos cultivos hortícolas, como ha sido observado en tomate (Solanum lycopersicum), lechuga (Lactuca sativa L.), ajo (Allium sativum L.) y frutilla (Fragaria vesca L.), entre otras especies (Domínguez et al., 2010).

Considerando la falta de datos y recomendaciones sobre dosis de estiércol vacuno y humus de lombriz a aplicar en el cultivo de lechuga, en este trabajo se evaluó el efecto de diferentes dosis de fertilización orgánica con el estiércol vacuno y humus de lombriz y su influencia en la producción. 


\section{METODO}

El experimento se llevó a cabo entre los meses de septiembre a noviembre del 2017, en el local del Programa de Investigación de Cultivos Olericolas (PICO) del Centro de Investigación Hernando Bertoni (CIHB), dependiente del Instituto Paraguayo de Tecnología Agraria (IPTA), Caacupé, departamento de Cordillera, ubicado sobre la ruta $2, \mathrm{~km} 48.5$, entre las coordenadas geográficas $25^{\circ} 23^{\prime} 16.38^{\prime \prime}$ latitud Sur y 57¹1'22.24" longitud Oeste, a 228 msnm.

Se utilizó lechuga de la variedad Graciela (tipo Pirati) sembrada en bandejas de germinación de isopor de 128 celdas, con sustrato compuesto por una mezcla de estiércol vacuno y mantillo en proporciones iguales $1: 1$.
Previo al trasplante se procedió a la aplicación de los tratamientos en los tablones. Los tratamientos fueron diferentes dosis y dos enmiendas orgánicas. La superficie de cada unidad experimental fue de $2 \mathrm{~m} 2$, con 4 hileras de lechuga por cada unidad, con un distanciamiento de $0.25 \mathrm{~m}$ entre hileras y $0.25 \mathrm{~m}$ entre plantas. La distancia entre unidades experimentales fue de $0.50 \mathrm{~m}$. La superficie total del experimento fue de 78 $\mathrm{m} 2$ con 864 plantas. La aplicación de los tratamientos se realizó el 29/09/2017 y el trasplante el 01/10/2017.

Se utilizó el diseño en Bloques Completos al Azar, con ocho tratamientos y tres repeticiones, incluidos los testigos (Tabla 1).

Tabla 1. Tratamientos constituidos por la combinación de dosis y enmiendas orgánicas.

\begin{tabular}{|l|l|}
\hline Tratamientos & \multicolumn{1}{|c|}{ Descripción } \\
T1 (Testigo) & 0 kg.m2 de humus de lombriz \\
T2 & 0,4 kg.m2 de humus de lombriz \\
T3 & 0,6 kg.m2 de humus de lombriz \\
T4 & 0,8 kg.m2 de humus de lombriz \\
T5 (Testigo) & 0 kg.m2 estiércol vacuno \\
T6 & 2 kg.m2 estiércol vacuno \\
T7 & 4 kg.m2 estiércol vacuno \\
T8 & 6 kg.m2 estiércol vacuno \\
\hline
\end{tabular}

Fuente: Elaboración propia

Para el área útil fueron cosechadas y evaluadas 10 plantas de las dos hileras centrales por cada tratamiento y repetición, y se realizaron las siguientes determinaciones: altura final de la planta, midiendo desde la base del suelo hasta el ápice de la hoja; número de hojas, contando las hojas por planta, masa fresca comercial y peso de la raíz, utilizando una balanza electrónica de precisión; masa seca de la parte aérea y de la raíz, por secado en estufa a $62^{\circ} \mathrm{C}$ durante 72 horas y pesado en balanza electrónica de precisión. La cosecha se realizó el 25/11/2017.

Los datos fueron sometidos al análisis de varianza (ANAVA), evaluando las diferencias entre medias por la prueba de Tukey al 5\% de probabilidad de error. Los análisis estadísticos se realizaron con el programa Infostat. 


\section{RESULTADOS}

Tabla 2. Efecto de diferentes dosis de dos enmiendas orgánicas en los componentes de rendimiento de la lechuga. Caacupé, 2017.

\begin{tabular}{|c|c|c|c|c|c|c|c|}
\hline Trat. & $\begin{array}{l}\text { Altura } \\
\text { de Planta } \\
\text { (cm) }\end{array}$ & $\begin{array}{l}N^{0} \text { de } \\
\text { Hojas }\end{array}$ & $\begin{array}{c}\text { Masa } \\
\text { fresca } \\
\text { comercial } \\
\text { (g) }\end{array}$ & $\begin{array}{c}\text { Masa } \\
\text { fresca de } \\
\text { la hoja } \\
\text { (g) }\end{array}$ & $\begin{array}{c}\text { Masa } \\
\text { seca de } \\
\text { la hoja } \\
\text { (g) }\end{array}$ & $\begin{array}{l}\text { Peso de } \\
\text { la raíz } \\
\text { (g) }\end{array}$ & $\begin{array}{l}\text { Peso seco } \\
\text { de la raíz } \\
\text { (g) }\end{array}$ \\
\hline $\mathrm{H} 1$ & $26,05 \mathrm{a}$ & $13,97 \mathrm{a}$ & $0,14 \mathrm{a}$ & $0,13 \mathrm{a}$ & $0,01 \mathrm{a}$ & $0,01 \mathrm{a}$ & $0,0040 \mathrm{a}$ \\
\hline $\mathrm{H} 2$ & $24,82 \mathrm{a}$ & $14,17 \mathrm{a}$ & $0,15 \mathrm{a}$ & $0,14 \mathrm{a}$ & $0,01 \mathrm{a}$ & $0,01 \mathrm{a}$ & $0,0043 \mathrm{a}$ \\
\hline H3 & $24,22 \mathrm{a}$ & 13,43 a & $0,17 \mathrm{a}$ & $0,11 \mathrm{a}$ & $0,01 \mathrm{a}$ & $0,01 \mathrm{a}$ & $0,0043 \mathrm{a}$ \\
\hline $\mathrm{H} 4$ & $23,82 \mathrm{a}$ & $13,90 \mathrm{a}$ & $0,15 \mathrm{a}$ & $0,11 \mathrm{a}$ & $0,01 \mathrm{a}$ & $0,01 \mathrm{a}$ & $0,01 \quad \mathrm{a}$ \\
\hline E5 & $23,50 \mathrm{a}$ & $14,00 \mathrm{a}$ & $0,13 \mathrm{a}$ & $0,12 \mathrm{a}$ & $0,01 \mathrm{a}$ & $0,01 \mathrm{a}$ & $0,0043 \mathrm{a}$ \\
\hline E6 & $24,88 \mathrm{a}$ & $13,95 \mathrm{a}$ & $0,15 \mathrm{a}$ & $0,14 \mathrm{a}$ & $0,01 \mathrm{a}$ & $0,01 \mathrm{a}$ & $0,01 \quad \mathrm{a}$ \\
\hline E7 & $25,20 \mathrm{a}$ & $14,70 \mathrm{a}$ & $0,14 \mathrm{a}$ & $0,13 \mathrm{a}$ & $0,01 \mathrm{a}$ & $0,01 \mathrm{a}$ & $0,0035 \mathrm{a}$ \\
\hline E8 & $26,70 \mathrm{a}$ & $14,70 \mathrm{a}$ & $0,16 \mathrm{a}$ & $0,15 \mathrm{a}$ & $0,01 \mathrm{a}$ & $0,01 \mathrm{a}$ & $0,0040 \mathrm{a}$ \\
\hline $\mathrm{CV}(\%)$ & 18,25 & 11,11 & 35,53 & 28,02 & 24,76 & 43,32 & 24,02 \\
\hline
\end{tabular}

Fuente: Elaboración propia

Se pudo identificar una tendencia donde de hojas, presentaron los tratamientos con los valores más elevados para las variables la dosis más alta, $6 \mathrm{~kg} . \mathrm{m} 2$ de estiércol vaaltura de planta (gráf. 1), masa fresca co- cuno, y las dosis más bajas: 0 ; 0.4 y 0.6 mercial, masa fresca, masa seca y número kg.m2 de humus de lombriz.

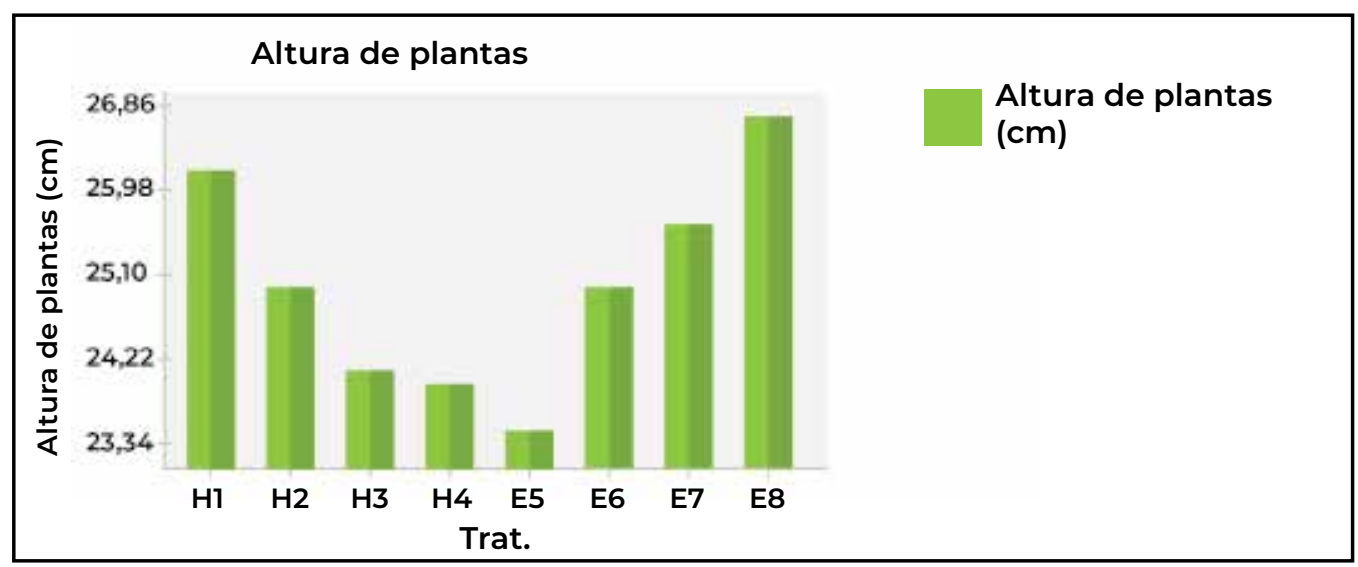

Fuente: Elaboración propia

Gráfico 1. Alturas de plantas en función a los tratamientos empleados

Así también, para las variables peso ra- de humus de lombriz, y las dosis bajas, 0 y dicular y peso seco radicular, los mayores $2 \mathrm{~kg} . \mathrm{m} 2$ de estiércol vacuno.

valores fueron la dosis más alta, $0,8 \mathrm{~kg} . \mathrm{m} 2$ 


\section{DISCUSIÓN}

Con respecto al humus de lombriz, Tombion et al. (2016) encontraron que el número de hojas, el peso fresco de la planta, sus partes constituyentes y el peso seco de la raíz fueron significativamente más elevados en plantas cultivadas con $20 \%$ de humus de lombriz, mientras que en el peso seco de la parte aérea y de la planta esta diferencia se observó también respecto al sustrato sin humus de lombriz.

Un efecto negativo de la incorporación de humus sobre el peso seco de raíces fue también observado por Paul \& Metzger (2005) en plantines de tomate (Solanum lycopersicum), pimiento (Capsicum annum L.) y berenjena (Solanum melongena L.) cultivados con $10 \%$ de humus, al compararlos con plantas obtenidas en un sustrato sin humus.

Las características físicas del sustrato también varían por la incorporación de humus de lombriz, como reportaron Melgar-Ramírez \& Pascual-Alex (2010) quienes, al comparar mezclas con proporciones crecientes de esta enmienda orgánica, observaron un aumento de la densidad aparente y una disminución de la porosidad total.

Ziech et al. (2014), encontraron que abonos orgánicos no presentaron diferencias significativas en cuanto a las características agronómicas de plantas de lechuga durante el primer ciclo, obteniéndose medias similares a la del testigo. También observaron que, en el segundo ciclo del cultivo, promovieron un aumento en el número de hojas, diferenciándose del testigo y del abono químico.

La adición de abonos orgánicos al suelo contribuye de acuerdo con el grado de descomposición y consecuente mineralización de esos residuos, interfiriendo directamente en la disponibilidad de nutrientes para las plantas, en especial para aquellas de ciclo corto, como la lechuga pudiendo presentas efectos inmediatos o residuales, influenciando en los cultivos subsecuentes (Peixoto Filho et al. 2013).

Mantovani et al. (2017) concluyeron que hubo un efecto significativo de la interacción entre las tasas de estiércol y los tipos de suelo sobre la altura de plantas de lechuga, rúcula y achicoria común, sobre la materia fresca y el rendimiento de materia seca de lechuga, rúcula y achicoria común, y sobre el rendimiento total de verduras de hoja, lo que indica que el efecto de la fertilización orgánica en estas propiedades depende del tipo de suelo. La fertilización orgánica con estiércol de ganado no alteró el diámetro del tallo de la lechuga. También observaron que los incrementos en el rendimiento de rúcula y achicoria común en respuesta a la fertilización orgánica fueron más marcados que para la lechuga, lo que indica que el efecto residual del abono de ganado en el rendimiento vegetal fue mayor que el efecto obtenido en el primer cultivo después de la fertilización orgánica. Esto se puede atribuir a la liberación lenta de $\mathrm{N}$ del estiércol del ganado, $\mathrm{y}$ al hecho de que las plantas de la primera cosecha explotaron el pozo de $\mathrm{N}$ del suelo.

En un experimento en maceta con suelo arenoso en un invernadero, Sampaio et al. (2007) encontraron que el estiércol del ganado causó la inmovilización del $\mathrm{N}$ del suelo en el primer mes después de la incorporación. Después de este período, la liberación de $\mathrm{N}$ aumentó progresivamente, alcanzando las cantidades más altas entre tres y seis meses después de la incorporación. Aun así, de acuerdo con estos autores, el efecto del estiércol en el suministro de nutrientes para las plantas de maíz fue más alto en el tercer o cuarto cultivo consecutivo.

En el campo, Peixoto Filho et al. (2013) 
sembraron cinco cultivos de lechuga posteriores, y reportaron un aumento en la productividad de esta hortaliza en respuesta a la fertilización orgánica, así como los efectos residuales de los abonos animales, en particular del estiércol de ganado, hasta la tercera cosecha. Además, según estos autores, la mineralización del estiércol de ganado fue más lenta que la del estiércol de pollo, lo que tiene una influencia directa en la liberación de nutrientes de los fertilizantes orgánicos a las plantas, especial- mente de cultivos con un ciclo más corto, como la lechuga.

Atendiendo todo lo mencionado anteriormente, se puede inferir que los tratamientos no presentaron diferencias significativas entre sí en este primer ciclo, pero podrían observarse esos efectos en ciclos posteriores, una vez que las enmiendas orgánicas aplicadas, estén convenientemente mineralizadas y con nutrientes liberados para provecho del cultivo.

\section{CONCLUSIONES}

En las condiciones en que se desarrolló el presente trabajo se concluye que:

La aplicación diferentes dosis de humus de lombriz y estiércol vacuna no ejercie- ron ningún efecto sobre los componentes de rendimiento y el rendimiento, considerando un ciclo del cultivo de lechuga.

\section{BIBLIOGRAFÍA}

1. Aalok, A; Tripathi, AK. \& Soni, P. (2008). Vermicomposting: A better option for organic solid waste management. Journal of Human Ecology, 24(1), 59-64.

2. Argüello, JÁ; Seisdedos, L; Díaz-Goldfarb, MC; Fabio, EA; Núñez, SB. \& Ledesma, A. (2013). Modificaciones anatomofisiológicas inducidas por residuos sólidos agrícolas (vermicompuesto) en plantines de lechuga (Lactuca sativa L.). Revista Internacional de Botánica Experimental, 82,289-295.

3. Basheer, $M$ \& Agrawal, OP. (2013). Effect of vermicompost on the growth and

productivity of tomato plant (Solanum lycopersicum) under field conditions. International Journal of Recent Scientific Research,4 (3), 247-249.

4. Batista, MAV; Vieria, LA; Sousa, JP; Freitas, JDB. \& Neto, FB. 2012. Efeito de diferentes fontes de adubação sobre a produção de alface no município de Igua-
tu-CE. Revista Caatinga, 25(3), 8-11.

5. Carrijo Azevedo Melo, L; Silva, CA. \& de Oliveira Dias, B. 2008. Caracterização da matriz orgânica de resíduos de origens diversificadas. Revista Brasileira de Ciência do Solo, 32(1).

6. DCEA (Dirección de Censos y Estadísticas Agropecuarias Py). (2016). Producción Agropecuaria: Síntesis Estadísticas. Zafra agrícola 2015-2016. San Lorenzo, Py, DCEA, 50 p.

7. Domínguez, J; Lazcano, C. \& Brandón, M. (2010). Influencia del vermicompost en el crecimiento de plantas. Aportes para la elaboración de un concepto objetivo. Acta Zoológica Mexicana, 26(2),359-371.

8. Fioreze, C; Ceretta, CA; Giacomini, SJ; Trentin, G; \& Lorensini, F. 2012. Liberação do $\mathrm{N}$ em solos de diferentes texturas com ou sem adubos orgânicos. Ciência Rural, 42(7). 
8. Grangeiro, LC, Costa, KD, Medeiros, MD, Salviano, AM., Negreiros, MD., Bezerra Neto, F. \& Oliveira, SD. (2006). Acúmulo de nutrientes por três cultivares de alface cultivadas em condições do Semi-Árido. Horticultura brasileira, 24(2), 190-194.

9. Mantovani, JR; Carrera, M; Lennon, J; Moreira, A; Marques, J; Bortolotti, A. \& Silva, DA. (2017). Fertility properties and leafy vegetable production in soils fertilized with cattle manure 1. 2125, 825-836.

10. Melgar-Ramírez, R. \& Pascual-Alex, MI. (2010). Characterization and use of a vegetable waste vermicompost as an alternative component in substrates for horticultural seedbeds. Spanish Journal of Agricultural Research, 8(4),1174-1182.

11. Paul, LC. \& Metzger, JD. (2005). Impact of vermicompost on vegetable transplant quality. HortScience, 40(7),2020-2023.

12. Peixoto Filho, JU; Freire, MBDS; Freire, FJ; Miranda, MF; Pessoa, LG. \& Kamimura, KM. (2013). Produtividade de alface com doses de esterco de frango, bovino e ovino em cultivos sucessivos. Revista Brasileira de Engenharia Agricola e Ambiental-Agriambi, 17(4).

13. Pôrto, ML, Alves, JDC, de Souza, AP, Araújo, RDC., \& de Arruda, JA. (2008). Nitrate production and accumulation in lettuce as affected by mineral nitrogen supply and organic fertilization. Horticultura Brasileira, 26(2), 227-230.
14. Sampaio, EDS; Oliveira, ND; \& Nascimento, PD. 2007. Eficiência da adubação orgânica com esterco bovino e com Egeria densa. Revista Brasileira de Ciência do Solo, 31, 995-1002.

15. Santi, A., Carvalho, M. A., Campos, O. R., da Silva, A. F., de Almeida, J. L. \& Monteiro, S. 2010. Ação de material orgânico sobre a produção e características comerciais de cultivares de alface. Hortic. bras, 28(1).

16. Silva, CA. 2008. Uso de resíduos orgânicos na agricultura. SANTOS, GA et al. Fundamentos da Matéria Orgânica do Solo. Porto Alegre: Metrópole, 597-621.

18. Tombion, L.; Puerta, AV.; Barbaro, LA; Miguel, A; Aires, B. \& Aires, B. (2016). Nota científica. Características del sustrato y calidad de plantines de lechuga (Lactuca sativa L.) según dosis de lombricompuesto. Scientific note. Substrate characteristics and lettuce (Lactuca sativa L.) seedling quality depending on the vermicompost, 46, 46-52.

19. Ziech, ARD; Conceição, PC; Luchese, AV.; Paulus, D. \& Ziech, MF. (2014). Cultivo de alface em diferentes manejos de cobertura do solo e fontes de adubação. Revista Brasileira de Engenharia Agrícola e Ambiental, 18(9), 948-954. 